\title{
Robust Unit Root Tests with an Innovation Variance Break
}

\author{
Yujin $\mathrm{Oh}^{1, a}$
}

${ }^{a}$ SKK GSB, Sungkyunkwan University

\begin{abstract}
A structural break in the level as well as in the innovation variance has often been exhibited in economic time series. In this paper we propose robust unit root tests based on a sign-type test statistic when a time series has a shift in its level and the corresponding volatility. The proposed tests are robust to a wide class of partially stationary processes with heavy-tailed errors, and have an exact binomial null distribution. Our tests are not affected by the size or location of the break. We set the structural break under the null and the alternative hypotheses to relieve a possible vagueness in interpreting test results in empirical work. The null hypothesis implies a unit root process with level shifts and the alternative connotes a stationary process with level shifts. The Monte Carlo simulation shows that our tests have stable size than the OLSE based tests.
\end{abstract}

Keywords: Unit root tests, robust sign test, structural break, break in variance.

\section{Introduction}

Since the seminal work of Perron (1990), a number of unit root tests with structural breaks have been developed. The presence of structural breaks leads to non-rejection of the null hypothesis of unit root and structural breaks are easily witnessed financial time series. Most existing unit root tests with breaks focus on the number of breaks, and on how to find the location of breaks, see inter alia, Banerjee et al. (1992), Zivot and Andrews (1992), Perron and Vogelsang (1992), Amsler and Lee (1995), Lumsdaine and Papell (1997), Clemente et al. (1998), Papell and Prodan (2003), and Lee and Strazicich (2003), Kapetanios (2005), and Cavaliere and Georgiev (2007). Contrary to the aforementioned literatures that mostly focused on the behavior of structural breaks in the level or trend of a time series, Hamori and Tokihisa (1997), Hsu (1977), and Kim et al. (2002) and Sen (2009) concentrate on the structural change in variance. Hamori and Tokihisa (1997), Hsu (1977), and Kim et al. (2002) consider a break only in the variance. Sen (2009) proposes unit root test with a contemporaneous break in the level and variance. The test statistic extends Perron's (1990) test using Kim et al.'s (2002) strategy based on a modified GLS transformation.

However, there is another important stylized feature of financial time series that should not go unnoticed: financial time series usually follow heavy-tailed distribution. We propose unit root tests with a contemporaneous break in the level and the variance. We extend So and Shin's (2001) methodology to a univariate time series with a break. Modifications and variations of the So and Shin (2001) methodology to other models are provided by, for example, Oh and So (2004) to panel model, Oh and So (2008) to cointegration test, and Shin et al. (2009) to panel model with cross sectional dependence. All of these unit root tests are robust to a class of heavy-tailed distributions.

The distributions of our test statistics under the null hypothesis are exactly binomial distribution so that exact test can be achieved. The asymptotics are standard normal distributions. Both the size

\footnotetext{
${ }^{1}$ SKK Graduate School of Business, Sungkyunkwan University, 53 Myungryun-dong 3-ga, Jongro-gu, Seoul 110-745, Korea. E-mail: ouj92@hotmail.com
} 
and the location of the breakpoints do not affect the distribution. This is a nice feature and a great contrast to most of existing literature having complex distributions under the null hypothesis. We also consider the breaks under the null and the alternative hypothesis. This allows the alternative hypothesis to unambiguously imply a stationary time series with a break.

The remainder of this paper is organized as follows. Section 2 proposes the test statistics and investigates the asymptotic null distributions of the tests. Section 3 provides the Monte Carlo simulation results and Section 4 concludes.

\section{Test Statistics}

We assume that the stochastic process $y_{t}$ has $T$ time series observations and is generated by the $\operatorname{AR}(1)$ process

$$
\left\{\begin{array}{l}
y_{t}=\mu_{t}+u_{t}, \\
u_{t}=\rho u_{t-1}+\sigma_{t} \epsilon_{t}, \quad t=1, \ldots, T,
\end{array}\right.
$$

where the initial value $y_{0}$ is a given (fixed or random) number; and $\mu_{t}=\mu$ if $t \leq T_{K}$ and $\mu_{t}=\mu^{*}$ otherwise. The $T_{K}$ stands for the break date. The $\sigma_{t}=\sigma$ if $t \leq T_{K}$ and $\sigma_{t}=\sigma^{*}$ otherwise; and $\epsilon_{t}$ satisfies the following Assumption 1.

Assumption 1. The $\left\{\epsilon_{t}\right\}_{t=1}^{T}$ is a sequence of errors which have zero conditional medians and have no atom at zero given $\mathcal{F}_{t-1}$ where $\mathcal{F}_{t}$ is a $\sigma$-field generated by $\left(y_{0}, y_{1}, \ldots, y_{t}\right)$.

We note that Assumption 1 admits a wide class of heavy-tailed errors. In this framework we are interested in testing for the null hypothesis that $H_{0}: \rho=1$ against the alternative hypothesis that $H_{1}:|\rho|<$ 1. In order to motivate the robust sign test, we first introduce a signed version of student's $t$-statistic for the case of no structural break. The usual $t$-statistic is $t=\sum_{t=1}^{T}\left(y_{t}-y_{t-1}\right)\left(y_{t-1}-\bar{y}\right) / \hat{\sigma}\left(\sum_{t=1}^{T}\left(y_{t-1}-\bar{y}\right)^{2}\right)^{1 / 2}$ where $\bar{y}=\sum_{t=1}^{T} y_{t} / T, \hat{\sigma}^{2}=\sum_{t=1}^{T}\left(\left(y_{t}-\bar{y}\right)-\hat{\rho}\left(y_{t-1}-\bar{y}\right)\right)^{2} /(T-1)$ and $\hat{\rho}=\sum_{t=1}^{T}\left(y_{t}-\bar{y}\right)\left(y_{t-1}-\bar{y}\right) / \sum_{t=1}^{T}\left(y_{t-1}-\right.$ $\bar{y})^{2}$. So and Shin (2001) substitute $\operatorname{sign}\left[\left(y_{t}-y_{t-1}\right)\left(y_{t-1}-\hat{\mu}_{t-1}\right)\right]$ for $\left(y_{t}-y_{t-1}\right)\left(y_{t-1}-\bar{y}\right)$ in the numerator of $t$, and then construct a new test statistic, $S=\sum_{t=1}^{T} \operatorname{sign}\left(y_{t}-y_{t-1}\right) \operatorname{sign}\left(y_{t-1}-\hat{\mu}_{t-1}\right)$, where $\hat{\mu}_{t}$ is the median of $y_{1}, \ldots, y_{t}$ and is $\mathcal{F}_{t}$-measurable. The median makes the test statistic invariant to monotone data transformation, and recursive method increases the power of the test statistic, see So and Shin (2001) for interesting finite sample properties of the test statistics such as the exact null distribution and the invariance to monotone data transformation. From this consideration, let $S_{T_{K}}^{(1)}$ be test statistic for the first regime,

$$
S_{T_{K}}^{(1)}=\sum_{t=2}^{T_{K}} \operatorname{sign}\left(y_{t}-y_{t-1}\right) \operatorname{sign}\left(y_{t-1}-\hat{\mu}_{t-1}\right)
$$

and $S_{T_{K}}^{(2)}$ be test statistic for the second regime,

$$
S_{T_{K}}^{(2)}=\sum_{t=T_{K}+2}^{T} \operatorname{sign}\left(y_{t}-y_{t-1}\right) \operatorname{sign}\left(y_{t-1}-\hat{\mu}_{t-1}^{*}\right),
$$

where $\hat{\mu}_{t}$ is the median of $y_{1}, \ldots, y_{t}$ and $\hat{\mu}_{t}^{*}$ is the median of $y_{T_{K}+1}, \ldots, y_{T}$. We introduce a robust sign test statistic, $t_{R}$, defined by

$$
t_{R}=S_{T_{K}}^{(1)}+S_{T_{K}}^{(2)} .
$$


Table 1: Size for normal distributions

\begin{tabular}{|c|c|c|c|c|c|c|c|c|c|c|c|}
\hline \multirow{2}{*}{$T$} & \multirow{2}{*}{$\lambda$} & & \multicolumn{9}{|c|}{$\sigma_{2} / \sigma_{1}$} \\
\hline & & & 4.00 & 2.50 & 1.67 & 1.25 & 1.00 & 0.80 & 0.60 & 0.40 & 0.25 \\
\hline \multirow{9}{*}{100} & \multirow{3}{*}{0.1} & $t_{R}$ & 0.043 & 0.041 & 0.042 & 0.045 & 0.042 & 0.041 & 0.044 & 0.042 & 0.045 \\
\hline & & $t_{p}$ & 0.046 & 0.045 & 0.044 & 0.048 & 0.051 & 0.055 & 0.067 & 0.112 & 0.223 \\
\hline & & $t_{s}$ & 0.053 & 0.053 & 0.051 & 0.052 & 0.053 & 0.053 & 0.053 & 0.054 & 0.057 \\
\hline & \multirow{3}{*}{0.3} & $t_{R}$ & 0.042 & 0.042 & 0.043 & 0.041 & 0.044 & 0.043 & 0.044 & 0.041 & 0.042 \\
\hline & & $t_{p}$ & 0.060 & 0.055 & 0.050 & 0.048 & 0.048 & 0.057 & 0.073 & 0.124 & 0.205 \\
\hline & & $t_{s}$ & 0.052 & 0.052 & 0.052 & 0.053 & 0.052 & 0.052 & 0.050 & 0.054 & 0.054 \\
\hline & \multirow{3}{*}{0.5} & $t_{R}$ & 0.040 & 0.041 & 0.041 & 0.040 & 0.042 & 0.040 & 0.041 & 0.043 & 0.040 \\
\hline & & $t_{p}$ & 0.114 & 0.086 & 0.060 & 0.054 & 0.050 & 0.052 & 0.063 & 0.083 & 0.110 \\
\hline & & $t_{s}$ & 0.053 & 0.052 & 0.049 & 0.055 & 0.051 & 0.053 & 0.053 & 0.053 & 0.053 \\
\hline \multirow{9}{*}{200} & \multirow{3}{*}{0.1} & $t_{R}$ & 0.050 & 0.048 & 0.049 & 0.051 & 0.050 & 0.051 & 0.049 & 0.050 & 0.049 \\
\hline & & $t_{p}$ & 0.046 & 0.045 & 0.047 & 0.048 & 0.049 & 0.055 & 0.070 & 0.112 & 0.232 \\
\hline & & $t_{s}$ & 0.053 & 0.051 & 0.052 & 0.052 & 0.050 & 0.050 & 0.053 & 0.049 & 0.052 \\
\hline & \multirow{3}{*}{0.3} & $t_{R}$ & 0.051 & 0.049 & 0.050 & 0.052 & 0.051 & 0.052 & 0.052 & 0.053 & 0.052 \\
\hline & & $t_{p}$ & 0.059 & 0.055 & 0.051 & 0.045 & 0.049 & 0.056 & 0.076 & 0.123 & 0.208 \\
\hline & & $t_{s}$ & 0.051 & 0.050 & 0.051 & 0.048 & 0.050 & 0.051 & 0.052 & 0.050 & 0.051 \\
\hline & \multirow{3}{*}{0.5} & $t_{R}$ & 0.050 & 0.048 & 0.051 & 0.052 & 0.049 & 0.050 & 0.051 & 0.050 & 0.051 \\
\hline & & $t_{p}$ & 0.118 & 0.086 & 0.065 & 0.054 & 0.050 & 0.053 & 0.063 & 0.085 & 0.116 \\
\hline & & $t_{s}$ & 0.052 & 0.052 & 0.052 & 0.053 & 0.051 & 0.051 & 0.052 & 0.051 & 0.053 \\
\hline
\end{tabular}

Note: $t_{R}$ denotes the proposed test; $t_{p}$ stands for Perron's test; and $t_{s}$ represents Sen's test

Now Assumption 1 enables us to identify the exact null distribution of the test statistics that is summarized in the following Theorem. Here $\Rightarrow$ stands for the convergence in distribution.

Theorem 1. Consider model (2.1) and assume $\left\{\epsilon_{t}\right\}$ satisfies Assumption 1. Then we have, under $H_{0}$, $\left(t_{R}+T-2\right) / 2 \sim B I N(T-2,0.5)$, and $t_{R} / \sqrt{T} \Rightarrow N(0,1)$, as $T \rightarrow \infty$.

Proof: (Theorem 1) Since $\left(S_{T_{K}}^{(1)}+T_{K}-1\right) / 2 \sim \operatorname{BIN}\left(T_{K}-1,0.5\right)$ under the null hypothesis for the first regime from Assumption 1, and $\left(S_{T_{K}}^{(2)}+T-T_{K}-1\right) / 2 \sim \operatorname{BIN}\left(T-T_{K}-1,0.5\right)$ we get the first result immediately. The second result follows directly from the Lindberg-Levy central limit theorem.

Our robust sign test statistic has several desirable properties. First, the exact binomial null distribution of the test statistic enables us to compute the $p$-value of the test for any finite $T$. Second, in contrast to other existing unit root tests, the test statistic is invariant to arbitrary monotone data transformations for each group. Third, we have no need to calculate the estimates for $\sigma$ and $\sigma^{*}$.

\section{Simulations}

In this section, we present the results of the Monte Carlo experiments to investigate the finite sample performance of the proposed tests. For the case of single break, we compare our tests with the Perron (1990), which is the baseline test statistic, and the Sen (2009) tests. We compare with Sen (2009) because this considers contemporaneous shifts in the level and variance but heavy-tailed errors are not allowed. We compare these statistics for the case of a structural break in the level with a contemporaneous variance shift and the case of a level shift with a homogeneous variance $\left(\sigma^{*} / \sigma=1.0\right)$.

We set $T=\{100,200\} ; \lambda=\{0.1,0.3,0.5\} ; T_{K}=[\lambda T]$; the $[x]$ indicates the greatest integer less than or equal to $x ; \mu=0, \mu^{*} \sim N(0,1) ; \sigma^{*} / \sigma=\{4,2.5,1.67,1.25,1,0.8,0.6,0.4,0.25\} ; y_{0}=0$; and $\epsilon_{t} \sim N(0,1)$. Once these parameters are generated independently of $\epsilon_{t}$, they fixed throughout replication and we replicate 10,000 times. Table 1 shows the sizes of the two test statistics at the 5\% level. 
Table 2: Size for heavy-tailed distributions

\begin{tabular}{|c|c|c|c|c|c|c|}
\hline$T$ & $\lambda$ & & $N(0,1) \rightarrow \operatorname{VM}(1,25)$ & $\mathrm{VM}(1,25) \rightarrow N(0,1)$ & $N(0,1) \rightarrow$ Cauchy & Cauchy $\rightarrow N(0,1)$ \\
\hline \multirow{6}{*}{100} & \multirow{3}{*}{0.3} & $t_{R}$ & 0.042 & 0.043 & 0.044 & 0.043 \\
\hline & & $t_{p}$ & 0.078 & 0.080 & 0.130 & 0.128 \\
\hline & & $t_{s}$ & 0.073 & 0.071 & 0.099 & 0.101 \\
\hline & \multirow{3}{*}{0.5} & $t_{R}$ & 0.043 & 0.043 & 0.045 & 0.046 \\
\hline & & $t_{p}$ & 0.075 & 0.072 & 0.117 & 0.119 \\
\hline & & $t_{s}$ & 0.072 & 0.067 & 0.126 & 0.105 \\
\hline \multirow{6}{*}{200} & \multirow{3}{*}{0.3} & $t_{R}$ & 0.053 & 0.055 & 0.051 & 0.049 \\
\hline & & $t_{p}$ & 0.067 & 0.067 & 0.136 & 0.135 \\
\hline & & $t_{s}$ & 0.061 & 0.059 & 0.115 & 0.107 \\
\hline & \multirow{3}{*}{0.5} & $t_{R}$ & 0.049 & 0.050 & 0.051 & 0.053 \\
\hline & & $t_{p}$ & 0.066 & 0.065 & 0.115 & 0.117 \\
\hline & & $t_{s}$ & 0.062 & 0.058 & 0.109 & 0.105 \\
\hline
\end{tabular}

Note: $t_{R}$ denotes the proposed test; $t_{p}$ stands for Perron's test; and $t_{s}$ represents Sen's test

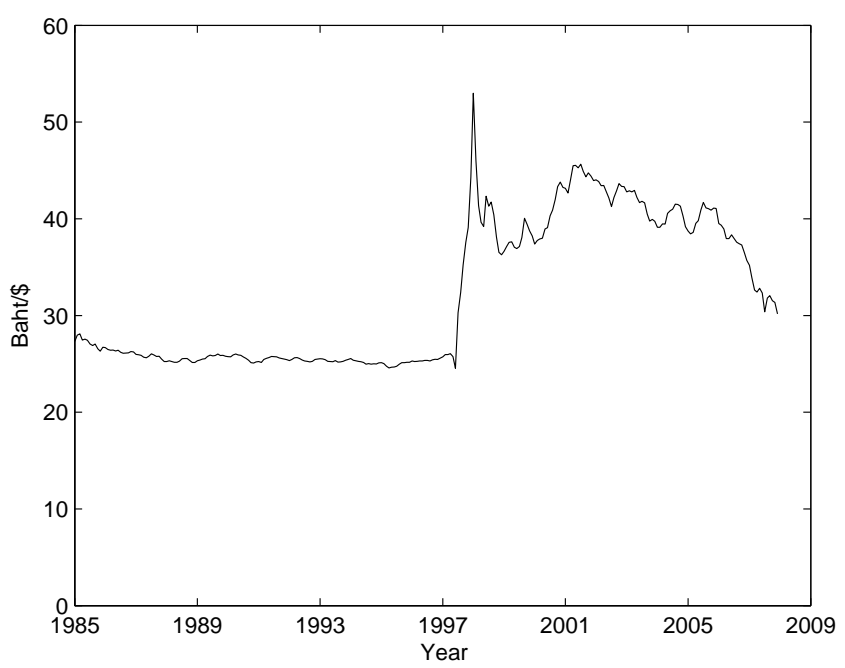

Figure 1: Thai Baht / US \$ Exchange Rate, 1985:01-2007:12

The simulation results show that the sizes of our test and Sen's test are stable for all cases. Meanwhile Perron's test shows distorted sizes when there are contemporaneous variance breaks.

We conduct another simulation for the case of heavy-tailed errors. A time series is divided in two parts and the first part follows a standard normal distribution and the second part follows $\operatorname{VM}(1,25)=$ $0.95 N(0,1)+0.05 N(0,25)$ or Cauchy distribution. The result is summarized in Table 2 .

Perron's test also shows unstable result and Sen's test statistic shows unstable result for this cases; however, our test statistic shows a stable size.

\section{An Empirical Illustration}

We apply the proposed test statistic to the data that is used in Sen (2009), the exchange rate between the Thailand Baht and the U.S. \$ using data over the period 1985:01-2007:12, see Figure 1.

The break date is specified as June 1997 in Sen (2009), and we follow this strategy. Perron's (1990) unit root statistic is equal to -4.0566 and results in mean-break stationary. Meanwhile Sen's (2009) unit root statistic is equal to -2.7003 and the null hypothesis cannot be rejected. Sen (2009) 
points out that allowing for the presence of a break in the innovation variance reverses the conclusion regarding the evidence of the presence of a unit root in the Baht $/ \$$ series. Our test statistic is equal to 0.3612 and the result is consistent with Sen's (2009). Our result indicates that the time series has unit root with break in the mean and variance.

\section{Concluding Remarks}

In this paper, we propose a sign-type unit root tests for a time series with level shifts contemporaneously in the mean and the volatility. The proposed tests are robust to a wide class of heavy-tailed errors. The distributions of the proposed tests under the null hypothesis are a binomial distribution and limiting distribution is a standard normal distribution. The distributions are not affected by the size and the location of the break.

In the Monte Carlo study, the proposed tests show stable size for heavy-tailed distributions; however, comparing test statistics show an unstable size for heavy-tailed errors.

\section{References}

Amsler, C. and Lee, J. (1995). An LM test for a unit root in the presence of a structural change, Econometric Theory, 11, 359-368.

Banerjee, A., Lumsdaine, R. L. and Stock, J. H. (1992). Recursive and sequential tests of the unit root and trend-break hypothesis: Theory and international evidence, Journal of Business and Economic Statistics, 10, 271-287.

Cavaliere, G. and Georgiev, I. (2007). Testing for unit roots in autoregressions with multiple level shifts, Econometric Theory, 23, 1162-1215.

Clemente, J., Montañés, A. and Reyes, M. (1998). Testing for a unit root in variables with a double change in the mean, Economics Letters, 59, 175-182.

Hamori, S. and Tokihisa, A. (1997). Testing for a unit root in the presence of a variance shift, Economics Letters, 57, 245-253.

Hsu, S. (1977). Tests for variance shift at an unknown time point, Journal of Applied Statistics, 26, 279-284.

Kapetanios, G. (2005). Unit root testing against the alternative hypothesis of up to $m$ structural breaks, Journal of Time Series Analysis, 26, 37-49.

Kim, T. H., Leybourne, S. and Newbold, P. (2002). Unit root tests with a break in innovation variance, Journal of Econometrics, 109, 365-387.

Lee, J. and Strazicich, M. C. (2003). Minimum LM unit root test with two structural breaks, Review of Economics and Statistics, 63, 1082-1089.

Lumsdaine, R. L. and Papell, D. H. (1997). Multiple trend breaks and the unit root hypothesis, Review of Economics and Statistics, 79, 212-218.

Oh, Y. and So, B. S. (2004). Robust tests for unit roots in heterogeneous panels, Economics Letters, 84, 35-41.

Oh, Y. and So, B. S. (2008). A new robust sign test for cointegration, Applied Economics Letters, 15, 971-974.

Papell, D. H. and Prodan, R. (2003). The uncertain unit root in US real GDP: Evidence with restricted and unrestricted structural change, Journal of Money, Credit and Banking, 36, 423-427.

Perron, P. (1990). Testing for a unit root in a time series with a changing mean, Journal of Business and Economic Statistics, 8, 153-162. 
Perron, P. and Vogelsang, T. J. (1992). Nonstationarity and level shifts with an application to purchasing power parity, Journal of Business and Economic Statistics, 10, 301-320.

Sen, A. (2009). Unit root tests in the presence of an innovation variance break that has power against the mean break stationary alternative, Statistics and Probability Letters, 79, 354-360.

Shin, D. W., Park, S. J. and Oh, M. S. (2009). A robust sign test for panel unit roots under cross sectional dependence, Computational Statistics \& Data Analysis, 53, 1312-1327.

So, B. S. and Shin, D. W. (2001). An invariant sign test for random walks based on recursive median adjustment, Journal of Econometrics, 102, 197-229.

Zivot, E. and Andrews, K. (1992). Further evidence on the great crash, the oil price shock, and the unit root hypothesis, Journal of Business and Economic Statistics, 10, 251-270. 